\title{
Within-day test-retest reliability of the 6-min walk test in patients with pulmonary fibrosis
}

To the Editor:

Interstitial lung diseases (ILDs) are a group of more than 150 different disorders characterized by inflammation of the lung parenchyma, or interstitium, often followed by the occurrence of pulmonary fibrosis. Patients with pulmonary fibrosis complain about exertional dyspnoea, exercise intolerance and reduced quality of life [1]. Poor exercise tolerance and oxygen desaturation during exercise are associated with poor survival [2]. Therefore, measuring exercise capacity in patients with pulmonary fibrosis is an important element of clinical management. The 6-min walk test (6MWT) is one of the most widely used clinical tests of functional exercise capacity in people with pulmonary fibrosis $[3,4]$.

The official European Respiratory Society (ERS)/American Thoracic Society (ATS) technical standard recommends that two 6MWTs should be performed as there is strong evidence of a learning effect on repetition [3]. Indeed, a mean improvement of $26.3 \mathrm{~m}$ during the second 6MWT has been found in patients with chronic obstructive pulmonary disease (COPD) [5]. Whether, and to what extent, the 6MWT is reproducible in patients with pulmonary fibrosis has been studied scarcely and, in one example, KozU et al. [6] reported a mean improvement of only $11 \mathrm{~m}$ during the second 6MWT in 35 patients with idiopathic pulmonary fibrosis (IPF). Indeed, performing the 6MWT twice in 1 day may prove too burdensome for the patient and too time consuming as, during a 1-day visit to a specialized ILD clinic, patients are already asked to undergo routine clinical tests (such as spirometry and chest X-rays) and to have blood drawn. The aim of this study was to assess the test-retest reliability of the 6MWT when performed within 1 day in patients with pulmonary fibrosis.

Data were collected in a prospective clinometric validation study that took place at the ILD Centre of Excellence, St. Antonius Hospital, Nieuwegein, The Netherlands, from June to December 2015 (Medical Research Ethics Committees United, NL51679.100.15/PT-PF). Eligible for participation were patients aged 18 years or older with a confirmed pulmonary fibrosis diagnosis according to the diagnostic criteria laid out in the ERS/ATS consensus statement [1]. Subjects were excluded if they had known cardiovascular or musculoskeletal disease that would prevent them from completing the 6MWT so as to conform to the official ERS/ATS technical standard [3]. Statistical analysis was performed using IBM SPSS Statistics (Version 23). The correlation of the distance walked in the 6MWT between test and retest was calculated by intraclass correlation coefficient (ICC) of single measures and absolute agreement with random effect ( $95 \% \mathrm{CI} ; \mathrm{p}<0.05)$.

Before the start of the first $6 \mathrm{MWT}$, blood pressure (BP; $\mathrm{mmHg}$ ), heart rate (HR; beats $\mathrm{min}^{-1}$ ), transcutaneous oxygen saturation $\left(\mathrm{SpO}_{2} ; \%\right.$ measured by pulse oximetry using a Nellcor-N-20 pulse oximeter (Medtronic, Minneapolis, MN, USA)), body mass index (BMI; $\mathrm{kg} \cdot \mathrm{m}^{-2}$ ) and body fat percentage (BFP; measured using an Omron HBF-306 body fat monitor (Omron Corp., Kyoto, Japan)) were assessed. Additionally, the medical research council (MRC) ratings [7] for situational perception of breathlessness were obtained.

Subjects performed the 6MWT according to the official ERS/ATS technical standards [3]. In brief, the $6 \mathrm{MWT}$ took place in a quiet 30 -m corridor and only standardised instructions and encouragement were given. It was performed twice in 1 day with the interval between tests being a minimum of $1 \mathrm{~h}$ and with each test starting at baseline values of heart rate (HRrest) and oxygen saturation $\left(\mathrm{SpO}_{2}\right.$ Rest). Each test was supervised by one of two trained physiotherapists (who walked slightly behind the subjects to avoid setting the walking pace) and, if present, oxygen therapy was delivered at a constant flow rate during both tests.

@ERSpublications

One 6-min walk test may not be enough to obtain the best 6-min walk distance in patients with pulmonary fibrosis http://ow.ly/cVfK3082RIL

Cite this article as: Bloem AEM, Veltkamp M, Custers JWH, et al. Within-day test-retest reliability of the 6-min walk test in patients with pulmonary fibrosis. Eur Respir J 2017; 49: 1601907 [https://doi.org/ 10.1183/13993003.01907-2016]. 
Both $\mathrm{SpO}_{2}$ and $\mathrm{HR}$ were monitored continuously during the test (telemetry), including after test end to determine the degree of heart rate recovery (HRR). Patients were not stopped during the test when $\mathrm{S}_{\mathrm{pO}_{2}}$ was below $80 \%$, as this also occurs during daily activities [8].

The primary outcome was the distance covered in 6 min $(6 \mathrm{MWD} ; \mathrm{m})$ while secondary outcomes included the lowest level of oxygen saturation $\left(\mathrm{SpO}_{2} \mathrm{Low}\right)$, the peak heart rate (HRpeak), the chronotropic response (CR; where $\mathrm{CR}=\mathrm{HR}$ peak-HRrest), and the degree of heart rate recovery between end of test and 1 min into recovery (HRR1; where $H_{R R}=H_{R}$ end-HRend+1) [9]. Ratings for dyspnoea and leg fatigue (using a modified 0-10 Borg scale) were also obtained before and after each 6MWT [10].

The sample consisted of 51 pulmonary fibrosis patients with a median age of 68 years (interquartile range: 63-74), of which $73 \%$ were men. The patients' pulmonary fibrosis diagnoses were divided between IPF (37\%), pulmonary fibrosis other than IPF (47\%) and unclassified pulmonary fibrosis (16\%). Supplemental oxygen was used by 11 subjects $(22 \%)$ during their 6MWTs. Generally, the patients were slightly overweight (BMI 27.4 $\pm 4.6 \mathrm{~kg} \cdot \mathrm{m}^{-2}$; BFP $31 \pm 7 \%$ ) and had impaired lung function as indicated by forced vital capacity (FVC), forced expiratory volume in $1 \mathrm{~s}(\mathrm{FEV} 1)$ and diffusing capacity of the lung for carbon monoxide $(D \mathrm{LCO})$ predictions $(\mathrm{FVC}($ predicted $)=79.7 \pm 21.5 \% ; \mathrm{FEV} 1$ ( predicted $)=81.5 \pm 21.4 \% ; \quad$ DLCO $($ predicted $)=46.1$ $\pm 16.4 \%)$. Moreover, $55 \%$ of patients endorsed the MRC dyspnoea scale at grade 3 while mean HRrest was 76

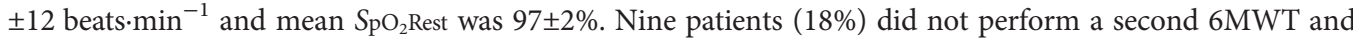
were therefore excluded from the test-retest analysis. The mean $6 \mathrm{MWD}$ of these nine patients $(394 \pm 166 \mathrm{~m})$ was on average $57 \mathrm{~m}$ shorter than that of the remaining 42 patients $(\mathrm{p}=0.346)$.

The mean $6 \mathrm{MWD}$ of the test-retest group was $451 \pm 104 \mathrm{~m}(70 \pm 13 \%$ predicted $)$ during the first $6 \mathrm{MWT}$ and $460 \pm 105 \mathrm{~m}(71 \pm 14 \%$ predicted) during the second 6MWT. Of this group, 28 patients (67\%) improved their 6MWD during the second test with a mean change of $21 \pm 17 \mathrm{~m}$, while 14 patients (33\%) had a similar or a decreased 6MWD (mean change: $-17 \pm 19 \mathrm{~m}$ ). The improvement in 6MWD between the first test and the second was small but significant $(8.4 \pm 25 \mathrm{~m} ; \mathrm{p}<0.05)$ and test-retest reliability for $6 \mathrm{MWD}$ showed excellent agreement (ICC $0.97,95 \%$ CI 0.94-0.98; figure 1). Comparable results were found for various subgroups. For example, patients with IPF $(n=14 ; 4.5 \pm 31 \mathrm{~m})$, patients with other types of pulmonary fibrosis $(\mathrm{n}=28 ; 10.4 \pm 22 \mathrm{~m})$, those with $D \mathrm{LCO} \geqslant 50 \%(\mathrm{n}=16 ; 12.8 \pm 23 \mathrm{~m})$ and those with DLCO $<50 \%(n=26 ; 5.7 \pm 27 \mathrm{~m})$. A total of 25 patients $(60 \%)$ had an $\mathrm{SpO}_{2}$ Low of $\leqslant 88 \%$ during the first and second 6MWTs. Furthermore, no significant differences were found between the first and second 6MWT for $\mathrm{SpO}_{2}$ Low $(85 \pm 8 \%$ versus $85 \pm 7 \%)$, HRpeak $\left(127 \pm 20\right.$ versus $131 \pm 19$ beats. $\left.\mathrm{min}^{-1}\right)$, CR $(49 \pm 20$ versus $51 \pm 19)$, HRR1 ( $14 \pm 17$ versus $10 \pm 17$ beats. $\mathrm{min}^{-1}$ ), or Borg symptom scores (at end of 6MWT) for dyspnoea (4.5 \pm 2.1 versus $4.6 \pm 2.2$ points) and fatigue (3.0 \pm 2.1 versus $3.3 \pm 2.3$ points).

The purpose of this study was to determine the test-retest reliability of the 6MWT when performed twice within the same day in patients with pulmonary fibrosis who visited a specialized outpatient ILD clinic. There are two main findings: 1) one in six pulmonary fibrosis patients was not able to walk the second $6 \mathrm{MWT}$ within the same day; 2) the 6MWT showed excellent test-retest reliability in the current sample of patients with pulmonary fibrosis. In the ERS/ATS technical standard [3], it is recommended to perform two 6MWTs in patients with chronic lung disease. However, this recommendation is mainly based on data from studies enrolling patients with COPD [5]. The current study shows a mean improvement of $8 \mathrm{~m}$ during the second $6 \mathrm{MWT}$, which corroborates the data of Kozu et al. [6]. While this difference is statistically significant, it clearly does not exceed the suggested minimal important difference for the 6MWD in patients with pulmonary fibrosis, which ranges between 21.6 and $30.5 \mathrm{~m} \mathrm{[5]}$, and the current findings obviously need to be corroborated by others.

FIGURE 1 Bland-Altman plot of the 6-min walk distance (6MWD). The mean difference and upper and lower limits of agreement $(95 \% \mathrm{Cl})$ are shown.

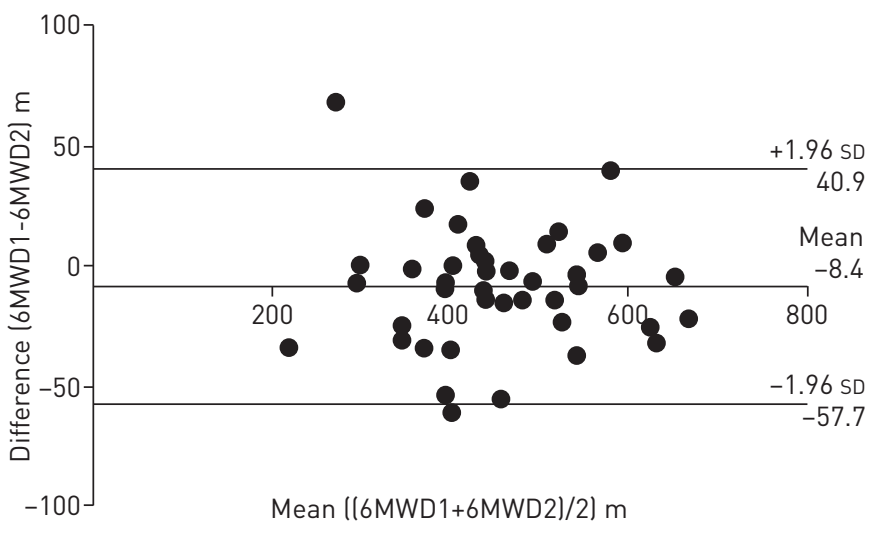


Interestingly, nine patients (18\%) were not able to perform the second 6MWT. Patients stated that the ability to perform only one $6 \mathrm{MWT}$ was due to both physical limitations (exhaustion, $\mathrm{n}=1$; knee pain, $\mathrm{n}=1$ ) and non-physical limitations (insufficient time for the second 6MWT, n=7). Without a second 6MWT we cannot be sure that we have obtained the best result. Then again, one $6 \mathrm{MWT}$ seems adequate to identify those patients with pulmonary fibrosis who suffer from exercise-induced oxygen desaturation, a variable of clinical interest as patients with this trait have a poor prognosis [2]. Indeed, $60 \%$ of the patients had an $\mathrm{SpO}_{2}$ Low value of $\leqslant 88 \%$ during the first $6 \mathrm{MWT}$ and no "new" exercise-induced desaturators were identified in the second 6MWT. In conclusion, these data suggest that one 6MWT may not be sufficient to obtain the best 6MWD in patients with pulmonary fibrosis. However, one 6MWT seems sufficient to identify those patients with pulmonary fibrosis who suffer from exercise-induced oxygen desaturation.

Ada E.M. Bloem ${ }^{1,2}$, Marcel Veltkamp ${ }^{2,3}$, Jan W.H. Custers ${ }^{1}$, Hanneke M. Dolk ${ }^{2}$, Jan C. Grutters ${ }^{2,3}$ and Martijn A. Spruit ${ }^{4,5,6}$

${ }^{1}$ Institute of Movement Studies, Faculty of Health Care, University of Applied Sciences Utrecht, Utrecht, The Netherlands. ${ }^{2}$ ILD Centre of Excellence, Dept of Pulmonology, St. Antonius Hospital, Nieuwegein, The Netherlands. ${ }^{3}$ Division of Heart and Lungs, University Medical Centre Utrecht, Utrecht, The Netherlands. ${ }^{4}$ Dept of Research and Education, Centre of Expertise for Chronic Organ Failure (CIRO), Horn, The Netherlands. ${ }^{5}$ The Centre for Rehabilitation Research (REVAL), Biomedical Research Institute (BIOMED), Faculty of Medicine and Life Sciences, Hasselt University, Diepenbeek, Belgium. ${ }^{6}$ Dept. of Respiratory Medicine, Maastricht University Medical Centre, NUTRIM School of Nutrition and Translational Research in Metabolism, Maastricht, The Netherlands.

Correspondence: Ada E.M. Bloem, Institute of Movement Studies, Faculty of Health Care, University of Applied Sciences Utrecht, Heidelberglaan 7, 3584 CS Utrecht, The Netherlands. E-mail: aadje.bloem@hu.nl

Received: June 282016 | Accepted after revision: Dec 122016

Conflict of interest: None declared.

\section{References}

1 Travis WD, Costabel U, Hansell DM, et al. An official American Thoracic Society/European Respiratory Society statement: update of the international multidisciplinary classification of the idiopathic interstitial pneumonias. Am J Respir Crit Care Med 2013; 188: 733-748.

2 Caminati A, Harari S. IPF: new insight in diagnosis and prognosis. Respir Med 2010; 104: Suppl. 1, S2-S10.

3 Holland AE, Spruit MA, Troosters T, et al. An official European Respiratory Society/American Thoracic Society technical standard: field walking tests in chronic respiratory disease. Eur Respir J 2014; 44: 1428-1446.

4 du Bois RM, Weycker D, Albera C, et al. Six-minute-walk test in idiopathic pulmonary fibrosis: test validation and minimal clinically important difference. Am J Respir Crit Care Med 2011; 183: 1231-1237.

5 Singh SJ, Puhan MA, Andrianopoulos V, et al. An official systematic review of the European Respiratory Society/ American Thoracic Society: measurement properties of field walking tests in chronic respiratory disease. Eur Respir J 2014; 44: 1447-1478.

6 Kozu R, Jenkins S, Senjyu H, et al. Peak power estimated from 6-minute walk distance in Asian patients with idiopathic pulmonary fibrosis and chronic obstructive pulmonary disease. Respirology 2010; 15: 706-713.

7 Kozu R, Jenkins S, Senjyu H. Evaluation of activity limitation in patients with idiopathic pulmonary fibrosis grouped according to Medical Research Council dyspnea grade. Arch Phys Med Rehabil 2014; 95: 950-955.

8 Park JH, Jegal Y, Shim TS, et al. Hypoxemia and arrhythmia during daily activities and six-minute walk test in fibrotic interstitial lung diseases. J Korean Med Sci 2011; 26: 372-378.

9 Swigris JJ, Olson AL, Shlobin OA, et al. Heart rate recovery after six-minute walk test predicts pulmonary hypertension in patients with idiopathic pulmonary fibrosis. Respirology 2011; 16: 439-445.

10 Borg G. Borg's Perceived Exertion and Pain Scales. Champaign, IL, Human Kinetics, 1998. 